\title{
Developments in the theory of collisionless reconnection in magnetic configurations with a strong guide field
}

\author{
F. Pegoraro ${ }^{1}$, D. Borgogno ${ }^{2}$, F. Califano ${ }^{1}$, D. Del Sarto ${ }^{1}$, E. Echkina ${ }^{3}$, D. Grasso ${ }^{2}$, T. Liseikina ${ }^{4}$, and F. Porcelli ${ }^{2}$ \\ ${ }^{1}$ Phys. Dept., University of Pisa and INFM, Pisa Italy \\ ${ }^{2}$ BPRG-INFM, Dept. of Energetics, Politecnico di Torino, Torino, Italy \\ ${ }^{3}$ Moscow State University, Moscow, Russia \\ ${ }^{4}$ Institute of Computational Technologies SD RAS, Novosibirsk, Russia
}

Received: 7 September 2004 - Revised: 9 November 2004 - Accepted: 12 November 2004 - Published: 19 November 2004

Part of Special Issue "Advances in space environment turbulence"

\begin{abstract}
We review some recent results that have been obtained in the investigation of collisionless reconnection in two dimensional magnetic configurations with a strong guide field in regimes of interest for laboratory plasmas. First we adopt a two-fluid dissipationless plasma model where the plasma evolution is described by the advection of two Lagrangian invariant fields. Then, we show that an analogous formulation in terms of Lagrangian invariants applies to the case where the electron response is obtained from a drift-kinetic model.
\end{abstract}

\section{Introduction}

Laboratory plasmas and space plasmas often exhibit different physical behaviour even in the case of closely related phenomena, such as magnetic field line reconnection, because of different parameter ratios and most importantly, because of different boundary conditions. In particular, laboratory plasmas are strongly constrained by periodicity conditions and, with the exception of Reverse Field Pinches, by the structural rigidity imposed by the externally generated and quasi-uniform toroidal magnetic field. On the other hand both types of plasmas are generally characterized by very large Lundquist numbers and, in particular for space plasmas, by conditions where the characteristic reconnection time is shorter than the electron-ion collision time. In such low collisionality regimes magnetic reconnection is made possible not by electron resistivity, but by electron inertia and by kinetic (thermal) effects.

Recently, collisionless magnetic field line reconnection in the laboratory has been re-examined in Ottaviani et al. (1993, 1995); Cafaro et al. (1998); Grasso et al. (2000, 2001); Porcelli et al. (2002); Del Sarto et al. (2003); Pegoraro et al.

Correspondence to: F. Pegoraro

(pegoraro@df.unipi.it)
(2004) and in Liseikina et al. (2004) with the aim of understanding the fast evolution time scales displayed in the experiments and of determining whether the magnetic island growth can saturate in the absence of dissipation. Of particular importance is the investigation of whether and in which form the reconnection time scales are determined by the microscopic spatial scales arising from electron inertial and thermal effects and that are involved in the reconnection process (see, e.g. Bhattacharjee et al., 2001; Shay et al., 2001; Wang et al., 2001; Fitzpatrick, 2004a,b). In addition, it is important to investigate whether the relative values of these microscopic spatial scales determine:

1) the final state of the saturated island and in particular the distribution of the current layers and of the fluid vorticity layers within the island,

2) the redistribution of the released magnetic energy among the different forms of plasma energy,

3) the onset of secondary instabilities driven by the reconnection process.

In this investigation the use of a two fluid model, first developed in Schep et al. (1994); Kuvshinov et al. $(1994,1998)$ has been particularly fruitful since, on the one hand, it contains the dispersive wave features of kinetic Alfvèn waves, features that are thought (Rogers et al., 2001) to play a fundamental role in all descriptions of collisionless reconnection, and on the other hand it exhibits elegant mathematical properties that allow us to describe the nonlinear plasma evolution in a rather compact and general form. The usefulness of such an approach has been evidenced also in the investigation of the kinetic regime performed in Liseikina et al. (2004), where it has been shown in that these mathematical properties can be extended to regimes where the electron response is derived from a drift kinetic equation (de Blank, 2001; Valori, 2001; de Blank et al., 2003). The extension of the investigation of the nonlinear evolution of magnetic reconnection 
to the kinetic regime is imposed by the fact that, in the presence of thermal effects, a fluid approach cannot be properly justified at the increasingly small scale lengths that are characteristic of the plasma nonlinear evolution in the absence of dissipation.

An important consequence of the results reviewed in this paper is that the physical process that occur at the microscopic scalelengths involved in the reconnection process determine its evolution in a very intrinsic way by changing the conservation laws that the plasma evolution must obey. This is very different from the case when magnetic reconnection occurs through the effect of electron resistivity, as in the latter case no invariant quantities are preserved.

\section{Magnetic connections}

Magnetic topology plays an important role in the global dynamics of high temperature plasmas. Within the ideal MHD plasma description, where

$$
\boldsymbol{E}+\boldsymbol{u} \times \boldsymbol{B} / c=0, \quad \Rightarrow \quad \frac{\partial \boldsymbol{B}}{\partial t}=\nabla \times(\boldsymbol{u} \times \boldsymbol{B}),
$$

with $\boldsymbol{E}(\boldsymbol{x}, t), \boldsymbol{B}(\boldsymbol{x}, t)$ the plasma electric and magnetic field and $\boldsymbol{u}(\boldsymbol{x}, t)$ the fluid plasma velocity, two plasma elements, separated by the vector $\delta \boldsymbol{l}(\boldsymbol{x}, t)$ that are initially connected by a magnetic field line $(\delta \boldsymbol{l} \times \boldsymbol{B}=0$ at $t=0)$, remain connected by a field line at any subsequent time, since $\delta \boldsymbol{l} \times \boldsymbol{B}$ is transported with the plasma (i.e., its Lie derivative vanishes)

$$
\frac{\partial(\delta \boldsymbol{l} \times \boldsymbol{B})}{\partial t}+(\boldsymbol{u} \cdot \nabla)(\delta \boldsymbol{l} \times \boldsymbol{B})=-(\nabla \boldsymbol{u}) \cdot(\delta \boldsymbol{l} \times \boldsymbol{B}) .
$$

This condition introduces a topological linking (magnetic connection) between plasma elements that is preserved during the ideal MHD plasma evolution. Magnetic linking constraints the plasma dynamics by making configurations with lower magnetic energy, but different topological linking, inaccessible.

Magnetic reconnection partially removes these constrains by allowing the field lines to decouple locally (i.e., around critical points) from the plasma motion and to reknit in a different net of connections. This localized breaking of the connections arises from physical effects neglected in Eq. (1) that are weak all over the plasma, but are locally enhanced by the formation of small spatial scales around critical points.

In collisionless magnetic field line reconnection, the decoupling between the magnetic field and the plasma motion occurs because of finite electron inertia (in the fluid limit) or thermal effects (in the kinetic plasma description). In the absence of dissipation, the plasma response both in the fluid and in the kinetic electron treatment admits generalized linking conditions that are preserved during the process of magnetic reconnection and that in a 2 -D configuration take the simple form of Lagrangian invariants.

\subsection{Generalized magnetic connections: an example}

In a collisionless cold plasma model the effect of electron inertia and of the Hall term in Ohm's law

$\boldsymbol{E}+\frac{\boldsymbol{u}}{c} \times \boldsymbol{B}=-\frac{m_{e}}{e} \frac{d \boldsymbol{u}_{e}}{d t}+\frac{1}{n e c} \boldsymbol{J} \times \boldsymbol{B}$,

can be accounted for by introducing the vector fields

$$
\begin{aligned}
& \boldsymbol{B}_{e} \equiv \boldsymbol{B}-\frac{m_{e} c}{e} \nabla \times \boldsymbol{u}_{e}=\nabla \times \boldsymbol{A}_{e}, \\
& \boldsymbol{E}_{e} \equiv \boldsymbol{E}+\frac{m_{e}}{2 e} \nabla u_{e}^{2}+\frac{m_{e}}{e} \frac{\partial \boldsymbol{u}_{e}}{\partial t}=-\nabla \varphi_{e}-\frac{1}{c} \frac{\partial \boldsymbol{A}_{e}}{\partial t},
\end{aligned}
$$

where subscripts $e$ denote electron quantities and $\boldsymbol{u}_{e}$ is the electron fluid velocity. The generalized vector potential $\boldsymbol{A}_{e}$ is proportional to the fluid electron canonical momentum and $\varphi_{e}$ to the total electron energy and reduce to vector potential $\boldsymbol{A}$ and to the electrostatic potential $\varphi$ in the limit of massless electrons. The vector fields $\boldsymbol{B}_{e}(\boldsymbol{x}, t)$ and $\boldsymbol{E}_{e}(\boldsymbol{x}, t)$ satisfy the homogeneous Mawxell's equations and the ideal Ohm's law in the form

$\boldsymbol{E}_{e}+\frac{\boldsymbol{u}_{e}}{c} \times \boldsymbol{B}_{e}=0$,

which leads to the generalized liking condition

$\frac{\partial\left(\delta \boldsymbol{l} \times \boldsymbol{B}_{e}\right)}{\partial t}+\left(\boldsymbol{u}_{e} \cdot \nabla\right)\left(\delta \boldsymbol{l} \times \boldsymbol{B}_{e}\right)=-\left(\nabla \boldsymbol{u}_{e}\right) \cdot\left(\delta \boldsymbol{l} \times \boldsymbol{B}_{e}\right)$.

Similarly, all the ideal MHD theorems (magnetic flux conservation, magnetic helicity conservation, linking number etc,) are recovered by substituting $\boldsymbol{B}_{e}$ for $\boldsymbol{B}$ and $\boldsymbol{u}_{e}$ for $\boldsymbol{u}$.

\subsection{Transitions between magnetic equilibria}

The breaking of the magnetic connections allows the system to access configurations with lower magnetic energy.

The possibility of a transition between two magnetic equilibria with different magnetic energies can be easily conceived in the case of dissipative reconnection, when the local decoupling between the magnetic field and the plasma motion is due to electric resistivity, $\boldsymbol{E}+\boldsymbol{u} \times \boldsymbol{B} / c=\eta \boldsymbol{J}$, since the excess magnetic energy that is released in the transition can be transformed into heat. The possibility of such a transition between two equilibrium states is less obvious in the nondissipative case where energy can only be transferred into mechanical or (reversible) internal energy so that one could expect that the system cannot be "stopped"in a new stationary equilibrium with a lower magnetic energy. Indeed this apparent difficulty is not very different from the one that occurs in the treatment of Landau damping in Vlasov's equation for the distribution function $f(\boldsymbol{x}, \boldsymbol{v}, t)$. In Vlasov's equation no energy is dissipated and particle-points (distribution elements) in phase space that lie initially on an $f=$ const hypersurface and that move along the characteristics of the single-particle Hamiltonian $H(\boldsymbol{x}, \boldsymbol{v}, t)$ lie at all times on an $f=$ const hypersurface (with the same value of the constant). This amounts to say that, in the absence of collisions, $f$-connections are preserved. 


\section{Two-dimensional configurations: connections and Lagrangian invariants}

The concept of magnetic connections simplifies in the case of two-dimensional (2-D) configurations where all quantities depend on $x, y$ and on time $t$ only. The magnetic configurations of interest here are characterized by a strong, externally imposed, $B_{z}$ field which is taken to be fixed and does not play the role of a dynamical variable and by an inhomogeneous shear field in the $\mathrm{x}-\mathrm{y}$ plane associated with a current density $J(x, y, t)$ along the z-axis. The field $B_{z}$ plays a very important physical role in determining the model that is appropriate to represent the plasma dynamics in the $\mathrm{x}-\mathrm{y}$ plane. Plasma configurations where $B_{z}$ is absent display a different behaviour both in the fluid and in the kinetic description.

In such a 2-D configuration, the magnetic and the electric field can be expressed as

$$
\begin{aligned}
\boldsymbol{B} & =B_{0} \boldsymbol{e}_{z}+\nabla \psi(x, y, t) \times \boldsymbol{e}_{z}, \\
\boldsymbol{E} & =-\nabla \varphi(x, y, t)+\frac{\boldsymbol{e}_{z}}{c} \frac{\partial \psi(x, y, t)}{\partial t},
\end{aligned}
$$

where the flux function $\psi(x, y, t)$ is the z-component of the vector potential of the shear magnetic field and $\varphi$ is the electrostatic potential which plays the role of the stream function for the plama motion in the $\mathrm{x}-\mathrm{y}$ plane. Then, the conserved connections between plasma elements moving in the $\mathrm{x}-\mathrm{y}$ plane take the form of Lagrangian invariants i.e., can be expressed in term of scalar quantities that are advected by the plasma motion and are constant along characteristics.

In the ideal MHD limit this Lagrangian invariant corresponds to the $\mathrm{z}$ component $A_{z}$ of the magnetic vector potential i.e. to the flux function $\psi$. Plasma elements that lie initially on a $\psi=$ const curve in the $\mathrm{x}-\mathrm{y}$ plane and that move along the characteristics of the stream function $\varphi$ remain at all times on the same $\psi=$ const curve, i.e., $\psi$-connections are preserved. Note that the conservation of a Lagrangian invariant is qualitatively different from that expressed e.g. by the continuity equation for the plasma energy density, insofar as the values of a Lagrangian invariant cannot be modified, but only redistributed.

\subsection{Cold fluid finite-mass electrons}

If the effect of electron inertia is included (in a cold electron fluid), the Lagrangian invariant corresponds to the $\mathrm{z}$ component $A_{z}-\left(m_{e} c / e\right) u_{e, z}$ of the "vector potential" of the field $\boldsymbol{B}_{e}$, which is proportional to the $\mathrm{z}$ component of the electron canonical fluid momentum. In most cases of interest for magnetic reconnection in a configuration with a strong $B_{z}$ field, the density perturbations can be taken to be small. Then, the term proportional to the $\mathrm{z}$ component $u_{e, z}$ of the electron velocity can be rewritten in terms of the $\mathrm{z}$ component of the electron current density $J$ (we disregard the ion motion along field lines). Within this approximation, denoting as customary $A_{z}-\left(m_{e} c / e\right) u_{e, z}$ by $F$, we have

$F(x, y, t)=\psi(x, y, t)-d_{e}^{2} \nabla^{2} \psi(x, y, t)$, with $-\nabla^{2} \psi(x, y, t) \equiv J(x, y, t)$ the $\mathrm{z}$ component of the current density and $d_{e} \equiv c / \omega_{p e}$ the collisionless electron skin depth. The Lagrangian invariant $F$ is advected by the stream function $\varphi$ of the electron motion in the $\mathrm{x}-\mathrm{y}$ plane which is proportional to the electrostatic potential according to

$$
\frac{\partial F}{\partial t}+[\varphi, F]=0,
$$

with the Poisson brackets $[f, g]$ defined by

$[f, g]=\mathbf{e}_{z} \cdot \nabla f \times \nabla g$.

This equation arises from the parallel component of Ohm's Eq. (3). The stream function $\varphi$ obeys the equation

$\frac{\partial U}{\partial t}+[\varphi, U]=[J, \psi]$,

where $U=\nabla^{2} \varphi$ is proportional to the plasma fluid vorticity and to the plasma density perturbation. This equation arises from the electron continuity equation and from the quasineutrality condition, after expressing the ion perturbed density in terms of the divergence of the ion polarization drift.

\subsection{Warm fluid finite-mass electrons}

When the effects of electron temperature are included, electron parallel compressibility leads to a modification of the conserved connections (Schep et al., 1994) and introduces a new microscopic scale-length $\rho_{s} \equiv\left(m_{e} / m_{i}\right)^{1 / 2} v_{\text {the }} / \Omega_{i}$ the so called ion-sound gyro-radius. When this contribution is included, an anisotropic electron pressure tensor appears in Ohm's law (as shown in Schep et al. (1994), this term is needed in order to compensate for a drift term in the electron inertia contribution). Then Eq. (11) becomes

$$
\frac{\partial F}{\partial t}+[\varphi, F]=\varrho_{s}^{2}[U, \psi],
$$

and the structure of the conserved connections is modified. By combining Eqs. $(13,14)$, we find that in this warm fluid finite-mass electron regime, two generalized connections are conserved which are expressed by the Lagrangian invariants $G_{ \pm}(x, y, t)$ defined by

$G_{ \pm}=\psi-d_{e}^{2} \nabla^{2} \psi \pm d_{e} \rho_{s} \nabla^{2} \varphi$,

that are advected by the generalized stream functions

$\varphi_{ \pm}=\varphi \pm\left(\rho_{s} / d_{e}\right) \psi$.

The new advection equations are

$\frac{\partial G_{ \pm}}{\partial t}+\left[\varphi_{ \pm}, G_{ \pm}\right]=0$.

Note the formal analogy with the standard 1-D VlasovPoisson problem for electrostatic Langmuir waves: the set of Eqs. (17) has the form of two coupled 1-D Vlasov equations, with $\mathrm{x}$ and $\mathrm{y}$ playing the role of the coordinate and of the conjugate momentum for the "distribution functions" $G_{ \pm}$of 
two "particle" species with opposite charges in the Poissontype equation for $\varphi$ and equal charges in the Yukawa-type equation for $\psi$

$$
\begin{aligned}
& d_{e} \rho_{s} \nabla^{2} \varphi=\frac{\left(G_{ \pm}-G_{ \pm}\right)}{2} \\
& \psi-d_{e}^{2} \nabla^{2} \psi=\frac{\left(G_{ \pm}+G_{ \pm}\right)}{2} .
\end{aligned}
$$

The stream functions $\varphi_{ \pm}$play the role of the single particle Hamiltonians.

\subsection{Dynamics: energy functional}

The dynamics of the plasma configuration is governed by the conserved energy functional $\mathcal{H}_{f l}(\psi, \varphi)^{1}$

$\mathcal{H}_{f l}(\psi, \varphi) \equiv \int \frac{d^{2} x}{2}\left(|\nabla \psi|^{2}+|\nabla \varphi|^{2}+d_{e}^{2} J^{2}+\varrho_{s}^{2} U^{2}\right)$.

The first term $\int d^{2} x|\nabla \psi|^{2} / 2$ represents the magnetic energy in the shear field, $\int d^{2} x|\nabla \varphi|^{2} / 2$ the plasma fluid kinetic motion, $\int d^{2} x d_{e}^{2} J^{2} / 2$ the energy of the ordered electron kinetic energy along field lines and $\int d^{2} x \varrho_{s}^{2} U^{2} / 2$ the work done by the parallel electron compression. This last term disappears in the limit of a cold electron fluid $\left(\varrho_{s} \rightarrow 0\right)$. Note that the magnetic energy and the plasma fluid kinetic motion, contain first derivatives or $\psi$ and $\varphi$ while the ordered electron kinetic energy and the work done by the parallel electron compression contain second derivatives, i.e., one can expect that the former two dominate at large scales and the latter two at small spatial scales. Note in addition that, in the absence of dissipative effects, there are no characteristic dissipative scalelengths in Eqs. (17) that can limit the nonlinear formation of small spatial scales. However we stress that, even when "resistive" or "viscous" effects are effectively included, they remain at all scales uniformly smaller than those of the electron inertial terms. This is guaranteed by the fact that fluid viscosity and electron resistivity do not introduce higher order differential operators in Eqs. (17) and that the characteristic evolution time is short on the the electron-ion collision time.

\section{Nonlinear reconnection regimes}

We are interested here in the nonlinear evolution of collisionless reconnection instabilities which arise because of the initial inhomogeneous current distribution in the $x-y$ plane.

\footnotetext{
${ }^{1}$ This energy functional is related to the possibility of describing Eqs. (17) in the form of Hamiltonian field equations with noncanonical variables and degenerate non-canonical Poisson brackets (Morrison (1984); Kuvshinov et al. (1994)). The kernel of these Poisson brackets is given by an infinite set of Casimirs defined as $\int d^{2} x \mathcal{C}_{ \pm}\left(G_{ \pm}\right)$with $\mathcal{C}_{ \pm}$arbitrary smooth functions.

By redefining the energy functional $\mathcal{H}_{f l}$ by adding the appropriate combination of Casimirs, we can rewrite $\mathcal{H}_{f l}$ in the form $\mathcal{H}\left(G_{ \pm}, \varphi_{ \pm}\right)=-\int d^{2} x\left(G_{+} \varphi_{+}+G_{-} \varphi_{-}\right) / 2$ (Kuvshinov et al., 1998).
}

This procedure is different from the one where forced magnetic reconnection is studied in configurations where magnetic flux is driven from their boundaries (in our case reconnection is "forced" by the initial conditions). As mentioned above, the decoupling between the plasma motion and the magnetic field occurs around critical points that correspond, in the 2-D configurations under examination, to the zeros of the shear field i.e., to the zeros of $\nabla \psi(x, y, t)$. As is customary for magnetic configurations of interest for laboratory plasmas, we consider initial configurations where critical points have degenerated into a critical line, i.e., initial configurations that depend only on one coordinate (say $\mathrm{x}$ ) and where the shear field vanishes along a line (the null line): $\psi_{0}=\psi_{0}(x)$ with $\partial \psi_{0}(x) / \partial x=\left.0\right|_{x=0}$.

The early development (linear phase) of the reconnection instabilities in such configurations has been thoroughly examined in the literature in terms of threshold conditions for the onset of the instabilities, growth rates and role of the boundary layer at the null line. In this layer a large current density cumulates and the topology of the shear field starts to be changed with the formation of magnetic islands bounded by magnetic separatrices. The interest here is to examine the nonlinear phase of a collisionless reconnection instability and the eventual saturation of the island growth. The threshold condition of the reconnection instabilities in null line configurations is controlled by the value of a parameter, usually denoted by $\Delta^{\prime}$, which "measures" both the total current that the instability drives in the boundary layer and the magnetic energy flux that is convected by the instability flows towards the null line. In the case of collisionless reconnection instabilities, where the decoupling between the magnetic field and the plasma occurs because of electron inertia, the regime that is of greatest interest is the so called large $\Delta^{\prime}$-regime ( $d_{e} \Delta^{\prime}$ of order unity).

The early nonlinear phase of the development of the collisionless reconnection instability in the cold electron limit was examined numerically and analytically in Ottaviani et al. $(1993,1995)$ and shown to lead to a narrow current layer along the initial null line and to a super-exponential phase with a reconnection rate, as measured by the reconnected flux $\delta \psi(t)$ at the island $\mathrm{X}$-point, larger than in the linear phase.

In order to investigate the long term nonlinear evolution and the "saturation" of a fast growing (large $d_{e} \Delta^{\prime}$ ) reconnection instability produced by electron inertia in a sheared magnetic equilibrium configuration with a null line, Eqs. (17) were integrated numerically in Cafaro et al. (1998); Grasso et al. (2000, 2001); Del Sarto et al. (2003) The scaling of the nonlinear evolution time with the microscopic parameters $d_{e}$ and $\varrho_{s}$ and the dependence of the spatial structure of the current density and vorticity distribution in the saturated phase on these parameters were of particular interest in such investigation.

\subsection{Numerical results: fluid regimes}

In this series of simulations periodic conditions were taken along y and the configuration parameters were chosen such 
that only one mode can be linearly unstable. The typical mesh sizes are $N_{x}=2048$ and $N_{y}=1024$. Random perturbations were imposed on the equilibrium configuration $\psi_{0}(x)=-L /\left[2 \cosh ^{2}(x / L)\right]$ in a simulation box with $L_{x}=2 L_{y}=4 \pi L$, taking $d_{e}=3 / 10 L$ and $\varrho_{s} / d_{e}$ in the range $0-1.5$. The accuracy of the integration was verified by testing the effects of numerical dissipation on the conservation of the energy and of the Lagrangian invariants.

\subsection{Formation of small spatial scales in the nonlinear phase}

The Lagrangian invariants $G_{ \pm}$differ from the flux function $\psi$ by the term $d_{e}^{2} J \pm d_{e} \varrho_{s} U$ which has small coefficients but involves higher spatial derivatives. As shown in Cafaro et al. (1998), magnetic reconnection proceeds unimpeded in the nonlinear phase because of the development near the $\mathrm{X}$ point of the magnetic island of increasingly small spatial scales that effectively decouple $\psi$ from $G_{ \pm}$. In Hamiltonian regimes the formation of such scales does not stop at some finite resistive scalelength. This corresponds to the formation of increasingly narrow current and vorticity layers. Because of the conserved $G_{ \pm}$connections, the spatial localization and structure of these layers depends on the value of $\varrho_{s} / d_{e}$.

\subsection{Mixing of the Lagrangian invariants and island growth} saturation

As mentioned above, in the reconnection model adopted, magnetic energy $\int d^{2} x|\nabla \psi|^{2}$ is transformed, in principle reversibly, into two forms of kinetic energy, one, $\int d^{2} x|\nabla \varphi|^{2}$, related to the plasma motion in the $x-y$ plane and one, $\int d^{2} x d_{e}^{2} J^{2}$, to the electron current along $\mathrm{z}$ and, for $\varrho_{s} \neq 0$, into electron parallel compression $\int d^{2} x \varrho_{s}^{2} U^{2}$. The last two energies involve quantities with higher derivatives. Being the system Hamiltonian, it is not a priori clear whether a reconnection instability can induce a transition between two stationary plasma configurations with different magnetic energies, as is the case for resistive plasma regimes where the excess energy is dissipated into heat.

Taking $\varrho_{s} / d_{e} \sim 1$, in Grasso et al. (2001) it was shown that, in spite of energy conservation, this transition is possible at a "macroscopic" level. A new coarse-grained stationary magnetic configuration can be reached because, as the instability develops, the released magnetic energy is removed at an increasingly fast rate from the large spatial scales towards the small scales that act a perfect sink. This allows the saturation of the island growth. Similarly, the constraints imposed by the conservation of the $G_{ \pm}$connections cease to matter at a macroscopic level.

The advection of the two Lagrangian invariants $G_{ \pm}$is determined by the stream functions $\varphi_{ \pm}$. The winding, caused by this differential rotation type of advection makes $G_{ \pm}$increasingly filamented inside the magnetic island, leading to a mixing process. These filamentary structures of $G_{ \pm}$do not influence the spatial structure of $\psi$ which remains regular. The advection of the Lagrangian invariants is shown in Fig. 1 where we draw the shaded isocontours of $G_{+}$at time
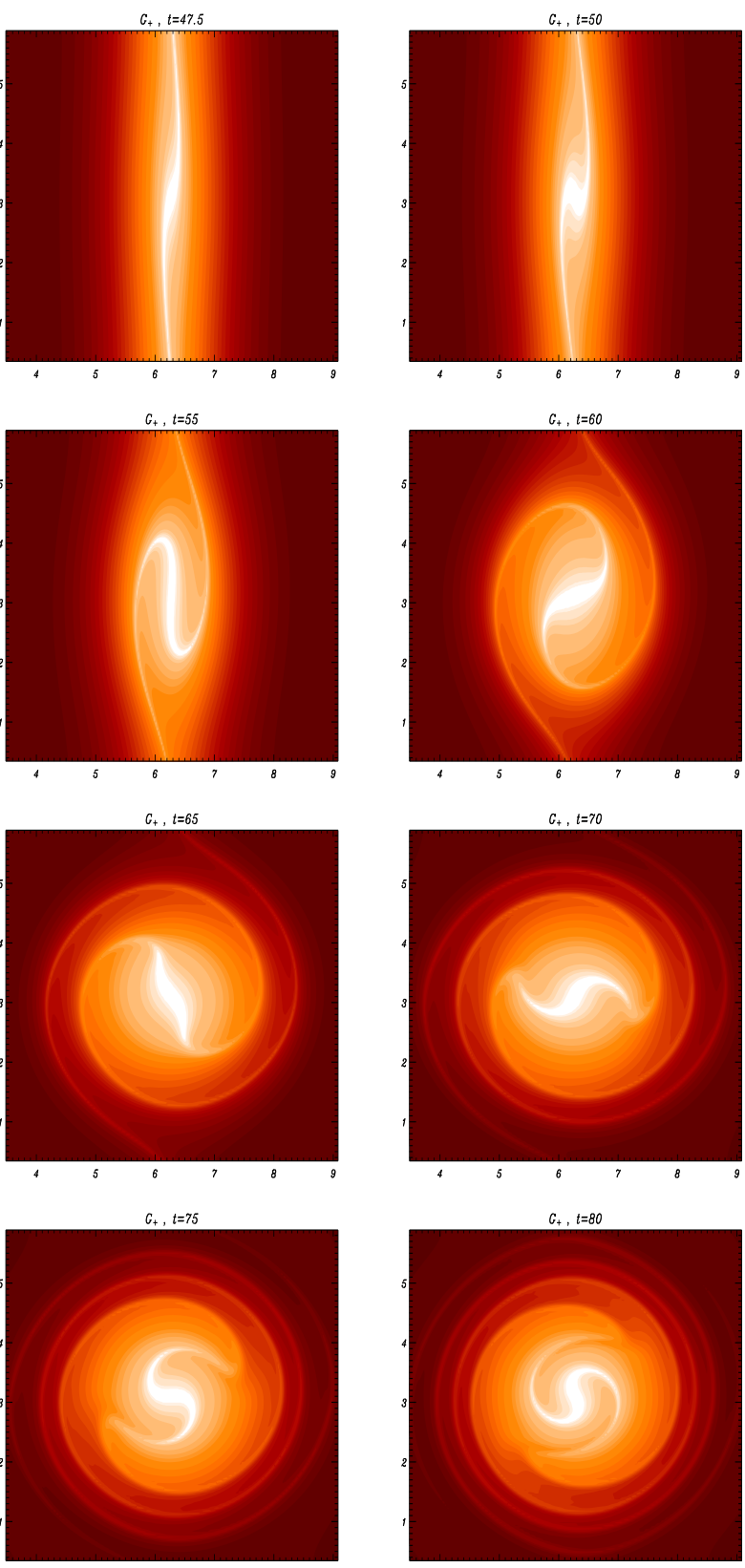

Fig. 1. Shaded isocontours of the Lagrangian invariant $G_{+}$in the central region of the $(\mathrm{x}, \mathrm{y})$ domain of integration. The frames are obtained at times $t=47.5,50,55,60,65,70,75,80$ for a run with $\varrho_{s} / d_{e}=1.5, L_{x}=4 \pi, L_{y}=21 \mathrm{pi}$.

$t=47.5,50,55,60,65,70,75,80$ for a run with $\varrho_{s} / d_{e}=1.5$. The analogy with the Bernstein-Greene-Kruskal (BGK) solutions of the Vlasov equation obtained in Manfredi (1993); Lancellotti et al. (1998); Brunetti et al. (2000); Lancellotti et al. (2003) for the nonlinear Landau damping of Langmuir waves was discussed in Grasso et al. (2001). Note that, as in the case of Vlasov equation, Hamiltonian reconnection naturally leads to the formation of elongated ribbons. Indeed, the conservation of $G_{ \pm}$in Eqs. (17) requires the formation 
of small scales, while the divergence free advection velocities derived from the stream functions $\varphi_{ \pm}$must necessarily stretch the plasma in order to preserve areas.

\subsection{Onset of a secondary Kelvin Helmholtz instability: tur-} bulent versus laminar mixing

The advection, and consequently the mixing, of the Lagrangian invariants can be either laminar or turbulent depending on the value of $\varrho_{s} / d_{e}$. The transition between these two regimes was shown in Del Sarto et al. (2003). to be related to the onset of a secondary Kelvin Helmholtz-type $(\mathrm{K}-\mathrm{H})$ instability driven by the velocity shear of the plasma motions that form because of the development of the reconnection instability. Whether or not the $\mathrm{K}-\mathrm{H}$ instability becomes active before the island growth saturates, affects the redistribution of the magnetic energy and determines whether a (macroscopically) stationary reconnected configuration is reached.

In the cold electron limit, $\varrho_{s} / d_{e}=0$, the system of Eqs. (17) becomes degenerate and the generalized connections are determined by a single Lagrangian invariant $F$. Initially, $F$ is advected along a hyperbolic pattern given by the stream function $\varphi$ which has a stagnation point at the $O$-point of the magnetic island. This motion leads to the stretching of the contour lines of $F$ towards the stagnation point and to the formation of a bar-shaped current layer along the equilibrium null line, which differs from the cross shaped structure found in the initial phase of the reconnection instability for $\varrho_{s} / d_{e} \neq 0$. Subsequently, $F$ contours are advected outwards in the $x$-direction. At this stage $F$ starts to be affected by a K-H instability that causes a full redistribution of $F$. In this phase the spatial structure of $F$ is dominated by the twisted filaments of the current density which spread through the central part of the magnetic island. The evolution of the Lagrangian invariant $F$ is shown in Fig. 2 where its shaded isocontours are plotted at times $t=100,102,106,111,117,127,137,147$ in the central region of the $(x, y)$ domain of integration.

After the onset of the secondary instability the contours of the vorticity $U$, see Fig. 3, exhibit a well developed turbulent distribution of monopolar and dipolar vortices, while those of $\psi$ remain regular although they pulsate in time. The energy balance shows that part of the released magnetic energy remains in the form of plasma kinetic energy corresponding to the fluid vortices in the magnetic island and that an oscillatory exchange of energy persists between the plasma kinetic energy and the electron kinetic energy (see also Bergmans et al., 1998) corresponding to the pulsations of the island shape.

This turbulent evolution of the nonlinear reconnection process persists in the non degenerate, finite electron temperature, case where the two Lagrangian invariants $G_{ \pm}$determine the generalized linking conditions However, as the ratio $\varrho_{s} / d_{e}$ is increased, i.e. as the electron temperature effects become more important, the onset of the K-H instability occurs later during the island growth and its effect on the current layer distribution becomes weaker. For $\varrho_{s} / d_{e} \sim 1$, no sign of
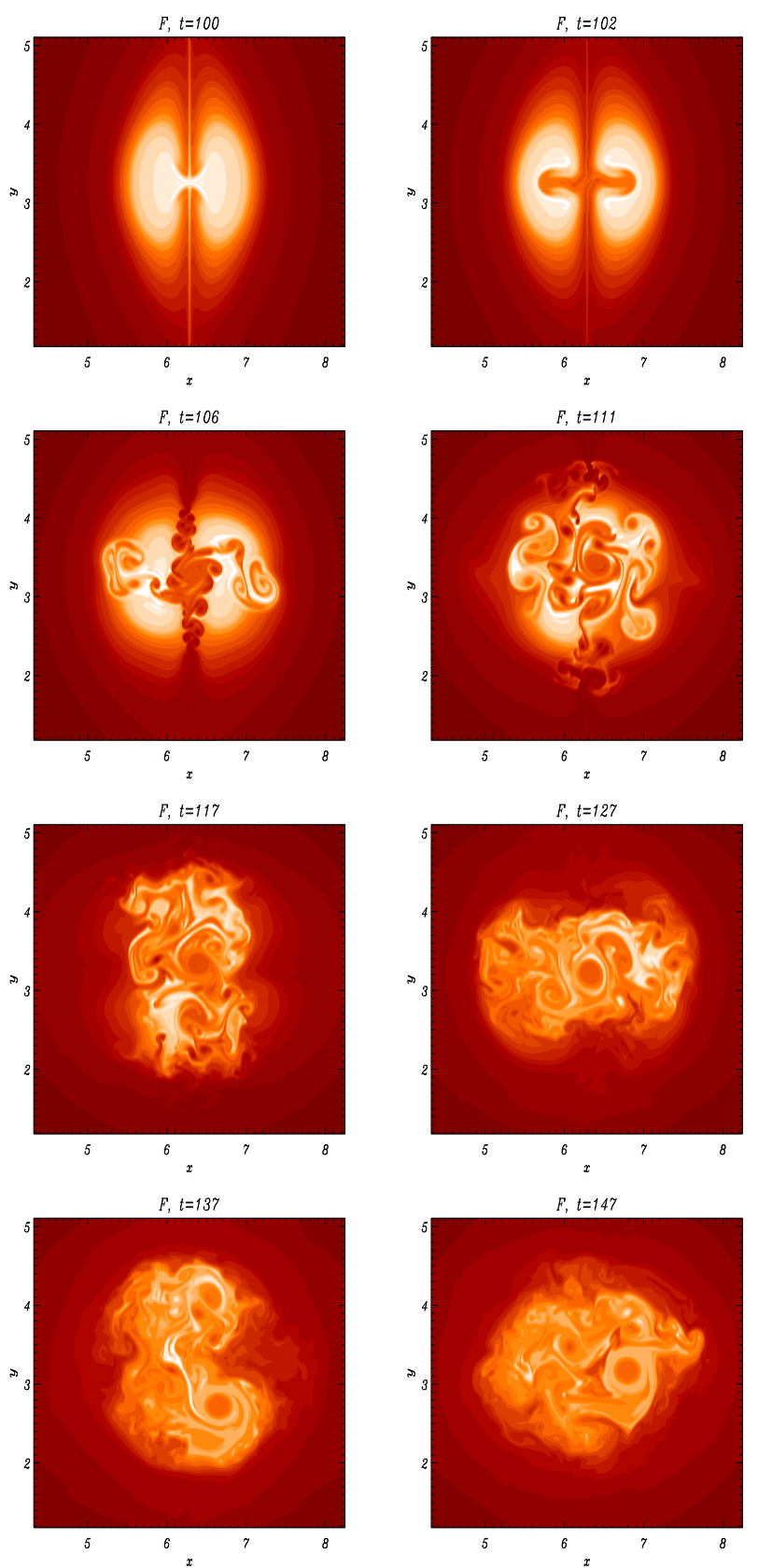

Fig. 2. Shaded isocontours of the Lagrangian invariant $F$ in the central region of the $(\mathrm{x}, \mathrm{y})$ domain of integration. The frames are obtained at times $t=100,102,106,111,117,127,137,147$ for a run with $\varrho_{s}=0, L_{x}=4 \pi, L_{y}=21 \mathrm{pi}$.

a secondary instability is detectable during the time the island takes to saturate its growth.

In the transitional regime, the advection pattern and the current layer structures exhibit an intermediate behaviour. Initially, $G_{ \pm}$are advected in opposite directions with a differential rotation, as is the case for $\varrho_{s} / d_{e}=1$. At later times they acquire features characteristic of the evolution of $F$ in the degenerate $\varrho_{s}=0$ case and their advection becomes K-H unstable leading to an almost turbulent distribution. 

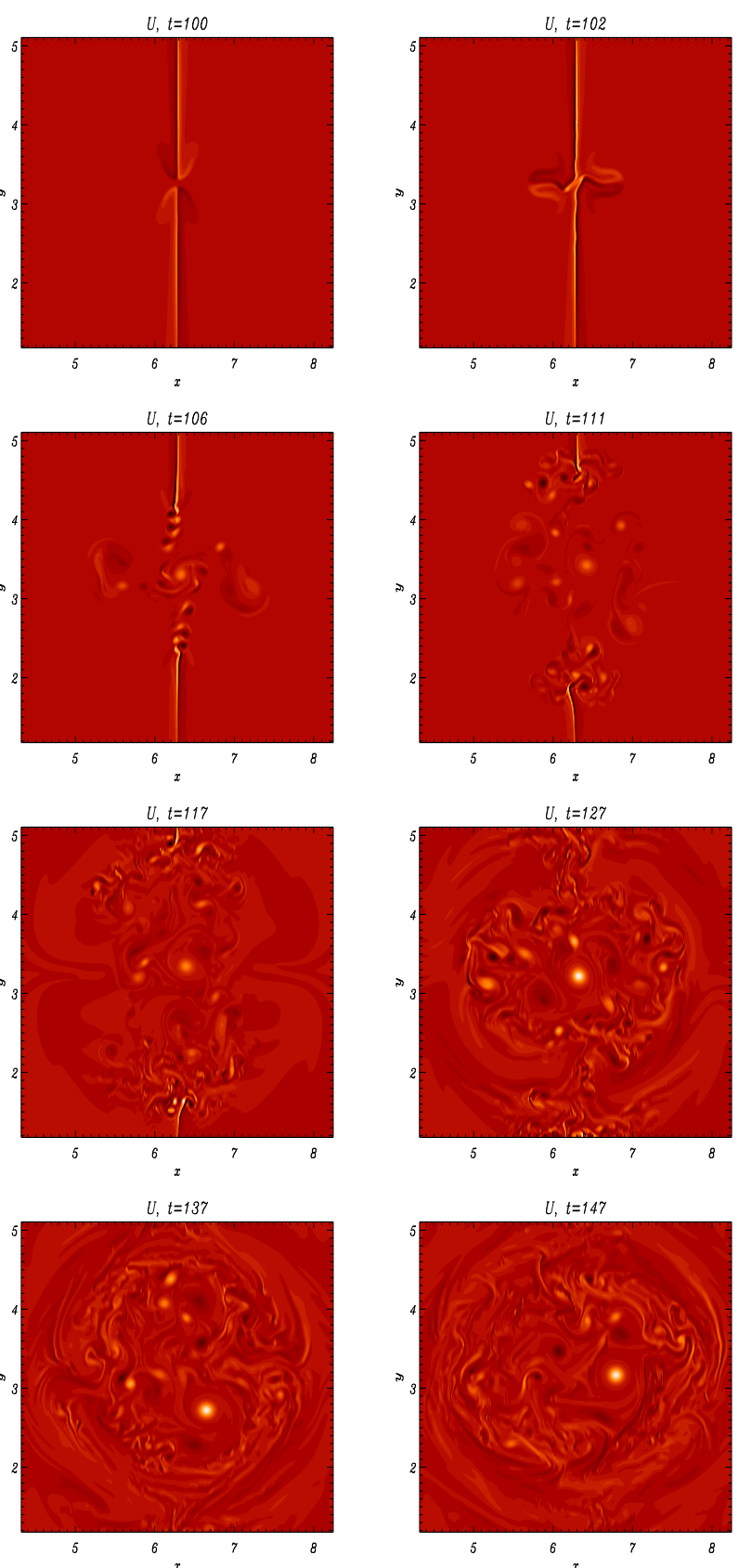

Fig. 3. Shaded isocontours of the vorticity $U$ in the central region of the $(\mathrm{x}, \mathrm{y})$ domain of integration. The frames are obtained at times $t=100,102,106,111,117,127,137,147$ for the same run of Fig. 2 .

\subsection{Need for a kinetic electron description}

The above results show that the conservation of the generalized connections in the reconnection process leads to the formation of current and vorticity layers with spatial scales that, in the absence of dissipation, becomes increasingly small with time. In this nonlinear phase of the development of the reconnection instability, in the presence of thermal effects, the fluid approximation becomes inconsistent inside the layers. The generalized connections and the constraints that they exert on the plasma dynamics apply to the case of a fluid plasma, where fluid elements can be defined and the linking property between fluid plasma elements can be formulated.

It thus becomes important to understand what is the role of the topological invariants in a kinetic electron description where e.g., the canonical momenta of the single electrons do not simply add up to give the fluid conserved Lagrangian invariant $F$ discussed above. The role of a finite electron temperature on the topological properties of the plasma is already evident from the above results, since the contribution of the parallel electron compressibility introduces two new Lagrangian invariants $G_{ \pm}$and two different streaming functions $\varphi_{ \pm}$instead of $F$ and $\varphi$, and consequently changes the nonlinear evolution of reconnection in a significant way.

\section{Drift kinetic formulation}

Let $\mathcal{F}\left(x, y, v_{\|}, t\right)$ be the drift-kinetic electron distribution function, with $v_{\|}$the electron velocity coordinate along field lines. It is convenient to adopt the electron canonical momentum, divided by the electron mass, $p_{\|}$, defined by ${ }^{2}$

$p_{\|} \equiv v_{\|}-\psi$,

as the kinetic variable instead of $v_{\|}$. Since we consider two dimensional ( $\mathrm{z}$ independent) fields and perturbations, $p_{\| \mid}$is a particle constant of the motion.

In the $x, y, p_{\|}, t$ variables the drift kinetic equation for the distribution function $f\left(x, y, p_{\|}, t\right) \equiv \mathcal{F}\left(x, y, v_{\|}, t\right)$ reads (de Blank, 2001; Valori, 2001; de Blank et al., 2003)

$\frac{\partial f}{\partial t}+\left[\varphi-\psi p_{\|}-\psi^{2} / 2, f\right]=0-\left[\varphi_{k i n}, f\right]$,

with $\varphi_{k i n}=\varphi-\psi p_{\|} / c-\psi^{2} / 2$. Note that in Eq. (21) the spatial derivatives are taken at constant $p_{\|}$and not at constant $v_{\|}$. For each fixed value of $p_{\|}$, the time evolution of $f$ corresponds to that of a Lagrangian invariant "density" advected by the velocity field obtained from the generalized stream function $\varphi_{k i n}$.

The advection velocity field is different on each $p_{\|}=$const foil. Thus, as noted in Liseikina et al. (2004), $f$ consists of an infinite number of Lagrangian invariants, each of them advected with a different velocity, that take the place of the two fluid invariants $G_{ \pm}$in Eqs. (17).

The fluid quantities are defined in terms of distribution function $f$ as follows

$\int d p_{\|} f\left(x, y, p_{\|}, t\right) n(x, y, t)$,

$\int d p_{\|} p_{\|} f\left(x, y, p_{\|}, t\right)[u(x, y, t)-\psi(x, y, t)] n(x, y, t)$,

\footnotetext{
${ }^{2} \mathrm{We}$ adopt the following normalizations $\varphi=e \varphi / m_{e} v_{\text {the }}^{2}$, $\psi=e \psi / m_{e} c v_{\text {the }}, x, y=x / L, y / L, t=t m_{e} v_{\text {the }}^{2} c / L^{2} e B_{0}, p_{\|}=$ $p_{\|} / v_{\text {the }}$, where $L$ is a characteristic length and the other symbols are standard.
} 
$\int d p_{\| \mid}\left[p_{\|}-u(x, y, t)+\psi(x, y, t)\right]^{2} f\left(p_{\| \mid}, x, y, t\right) \Pi_{||||}$,

where $n(x, y, t)$ and $u(x, y, t)$ are the normalized electron density and fluid velocity and $\Pi_{\|\|}(x, y, t)$ is the $(z, z)$ component of the pressure tensor. Then Ampere's equation reads

$d_{e}^{2} \nabla^{2} \psi=n u$

and, as in the fluid case, the ion equation of motion together with quasineutrality give

$\left(n-n_{0}\right)=\rho_{s}^{2} \nabla^{2} \varphi$,

where $n_{0}=n_{0}(x)$ is the initial normalized density and the density variations are supposed to remain small.

The above system of equations admits a conserved energy functional $\mathcal{H}_{\text {kin }}$

$\mathcal{H}_{k i n}=\int \frac{d^{2} x}{2}\left[d_{e}^{2}(\nabla \psi)^{2}+\rho_{s}^{2}(\nabla \varphi)^{2}+n u^{2}+\Pi_{||||}\right]$

Aside for the normalization, the main difference between these energy terms and the corresponding ones derived in the fluid case, see Eq. (19), is in the expression of the electron compression work, as natural in a kinetic theory, the pressure tensor $\Pi_{\|\| \mid}$cannot be expressed in terms of the lower order moments of the distribution function.

\subsection{Electron equilibrium distribution function}

The stationary solutions of Eq. (21) are of the form $f=f\left(p_{\|}, \varphi_{k i n}\right)$. Using the identity for the single particle energy

$v_{\|}^{2} / 2-\varphi=p_{\|}^{2} / 2-\varphi_{k i n}$,

we can write a stationary distribution function that depends only on the particle energy as $f=f\left(p_{\|}^{2} / 2-\varphi_{k i n}\right)$, while the well known static $\left(\varphi_{0}=0\right)$ Harris pinch equilibrium distribution (Harris (1962)) is given by ${ }^{3}$

$f=f_{0} \exp \left[-\left(p_{\|}^{2}-2 \varphi_{k i n}\right)-2 v^{*} p_{\|}\right]$

In order to have a less inhomogeneous plasma configuration we can add a pedestal (Maxwellian) distribution function of the form $f_{p e d}=f_{00} \exp \left[-\left(p_{\|}^{2}-2 \varphi_{k i n}\right)\right]$. The corresponding self consistent vector potential $\psi_{0}(x)$ is given by $\psi_{0}(x)=\left(1 / v^{*}\right) \ln (\cosh x)$ and the shear magnetic field has the standard hyperbolic tangent distribution.

\subsection{Evolution of the $p_{\|}=$const foils}

We write the distribution function with $f\left(x, y, t, p_{\|}\right)$as $f\left(x, y, t, p_{\|}\right)=\int d \bar{p}_{\| \mid} \delta\left(\bar{p}_{\| \mid}-p_{\|}\right) f\left(x, y, t, \bar{p}_{\|}\right)$. This is a foliation of the electron distribution function in terms of the infinite number of Lagrangian invariants obtained by taking the

\footnotetext{
${ }^{3}$ In velocity variable $v_{\|}$this distribution corresponds to $\mathcal{F}_{0} \exp \left[-\left(v_{\|}-v^{*}\right)^{2}-2 v^{*} \psi\right]$ and leads to a particle and current density of the form $n=n_{0} \exp \left(-2 v^{*} \psi\right)$ and $j=-n_{0} v^{*} \exp \left(-2 v^{*} \psi\right)$, where $j$ is normalized on $n_{o} e v_{\text {the }}$ and $v^{*}$ is the standard parameter related to the diamagnetic fluid motion.
}

distribution function $f$ at fixed electron canonical momentum. Within the drift-kinetic equation each $\bar{p}_{\|}$-foil evolves independently, while all foils are coupled through Maxwell's equations. The total number of particles in each foil is constant in time.

In the initial configuration, the spatial dependence of each $\bar{p}_{\|}$-foil is given for the case of the Harris distribution by $\exp \left(2 \bar{\varphi}_{k i n}\right)=\exp \left(-2 \psi \bar{p}_{\|}-\psi^{2}\right)=\exp \left[\bar{p}_{\|}^{2}-\hat{v}_{\|}(x)^{2}\right]$, where $\hat{v}_{\|}(x) \equiv v_{\|}\left(\psi, \bar{p}_{\|}\right)=\bar{p}_{\|}+\left(1 / v^{*}\right) \ln (\cosh x)$. For negative values of $\bar{p}_{\|}$the maximum of the argument of the exponent is located at $x= \pm \operatorname{arccosh}\left[\exp \left(-v^{*} \bar{p}_{\|}\right)\right]$i.e. the foil is localized in space within two symmetric bands, respectively to the right and to the left of the neutral line of the magnetic configuration. For positive values of $\bar{p}_{\|}$all the foils are centered around $x=0$.

\subsection{Nonlinear twist dynamics of the foils}

In the adopted drift kinetic framework the $\bar{p}_{\|}$-foils take the role of the Lagrange invariants $G_{ \pm}$of the fluid plasma description. In this perspective, the dynamics of the foils can be predicted by looking at the form of stream function $\varphi_{k i n}$ inside each foil. The advection velocity can be written as

$\boldsymbol{e}_{z} \times \nabla\left(\varphi-\psi p_{\|}-\psi^{2} / 2\right)=\boldsymbol{e}_{z} \times \nabla \varphi+\left(p_{\|}+\psi\right) \nabla \psi \times \boldsymbol{e}_{z}$,

which represents the particle $E \times B$ drift and their free motion along field lines.

At fixed $p_{\|}=\bar{p}_{\|}$we see that depending on the sign of $\psi+\bar{p}_{\|}=\hat{v}_{\|}(x)$, the advection velocity field takes two counter oriented rotation patterns, reminiscent of those that advect $G_{-}\left(G_{+}\right)$in fluid theory. In the equilibrium configuration where all quantities are function of $\psi=\psi(x)$ and $\varphi=0$, this advection corresponds to the free particle motion along $\psi=$ const surfaces inside each foil. However, when the instability starts to move the plasma along the $x$ axis and $\partial \varphi / \partial y \neq 0$, the portions of the foil where $\hat{v}_{\|}>0$ or where $\hat{v}_{\|}<0$ bend in opposite directions. This leads to a distortion and twist of the foils and to their eventual spatial mixing, analogously to the mixing of $G_{ \pm}$in fluid theory.

\section{Numerical results: drift-kinetic regime}

For a Harris equilibrium the evolution of the reconnection instability is characterized by three dimensionless parameters that can be expressed as the dimensionless ion sound gyroradius $\rho_{s}$ and the electron skin depth $d_{e}$ from Poisson's and Ampere's equations respectively, and $n_{0}$. In fact, when $d_{e}$ and $n_{0}$ are given, $v^{*}$, and thus $\psi_{0}$, are determined implicitly by the choice of $L$.

The size of the simulation box along $y$ has been chosen equal to $4 \pi$ such that the parameter $\Delta^{\prime}$ is positive only for the lowest order mode corresponding to $k_{y}=1 / 2$ so that only the $k_{y}=1 / 2$ mode can be linearly unstable. The simulation box is 40 long in the $x$ direction, with periodic boundary conditions in $\mathrm{y}$ and first type boundary conditions in $\mathrm{x}$. We have taken fixed $\rho_{s}=1$ and $d_{e}=1, v^{*}=4$, corresponding 

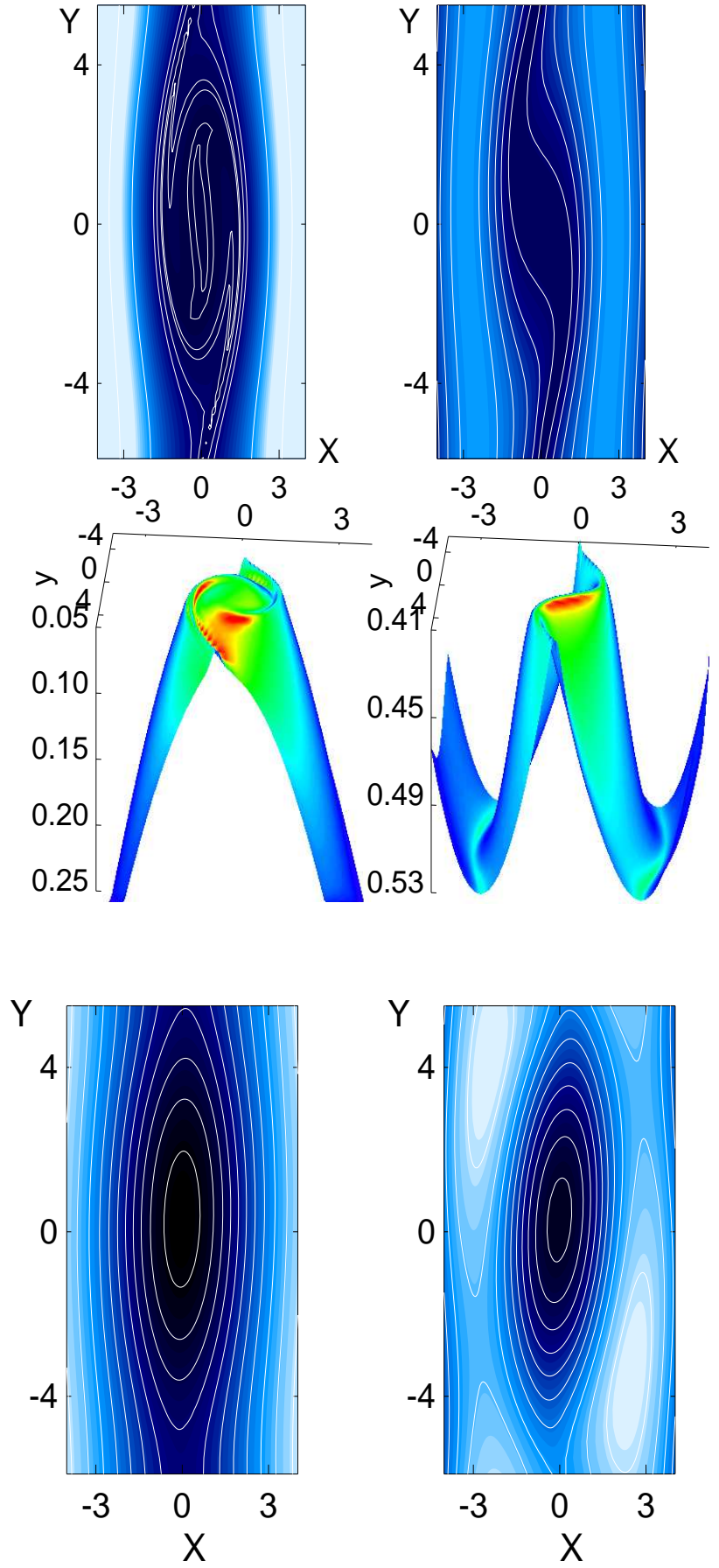

Fig. 4. Contour plots (top) and 3-D plots (middle) of the $p_{\|}=$constant foils of electron distribution function and contour plots (bottom) of the kinetic stream function $\varphi_{\text {kin }}$ at $t=81$ for $p_{\|}=-1.5,-0.5$ from left to right in the interval $-4<x<4$ around the neutral line. Note the different scales in the vertical axes of the 3-D plots.

to $\psi_{0}=1 / 4, n_{0}=1 / 16, d_{e}=1, v^{*}=2\left(=>\psi_{0}=1 / 2, n_{0}=1 / 4\right)$, $d_{e}=0.5, v^{*}=2\left(=>\psi_{0}=1 / 2, n_{0}=1 / 16\right)$. Smaller values of $v^{*}$ correspond to larger instability growth rates i.e., to faster evolving instabilities where the saturation of the island
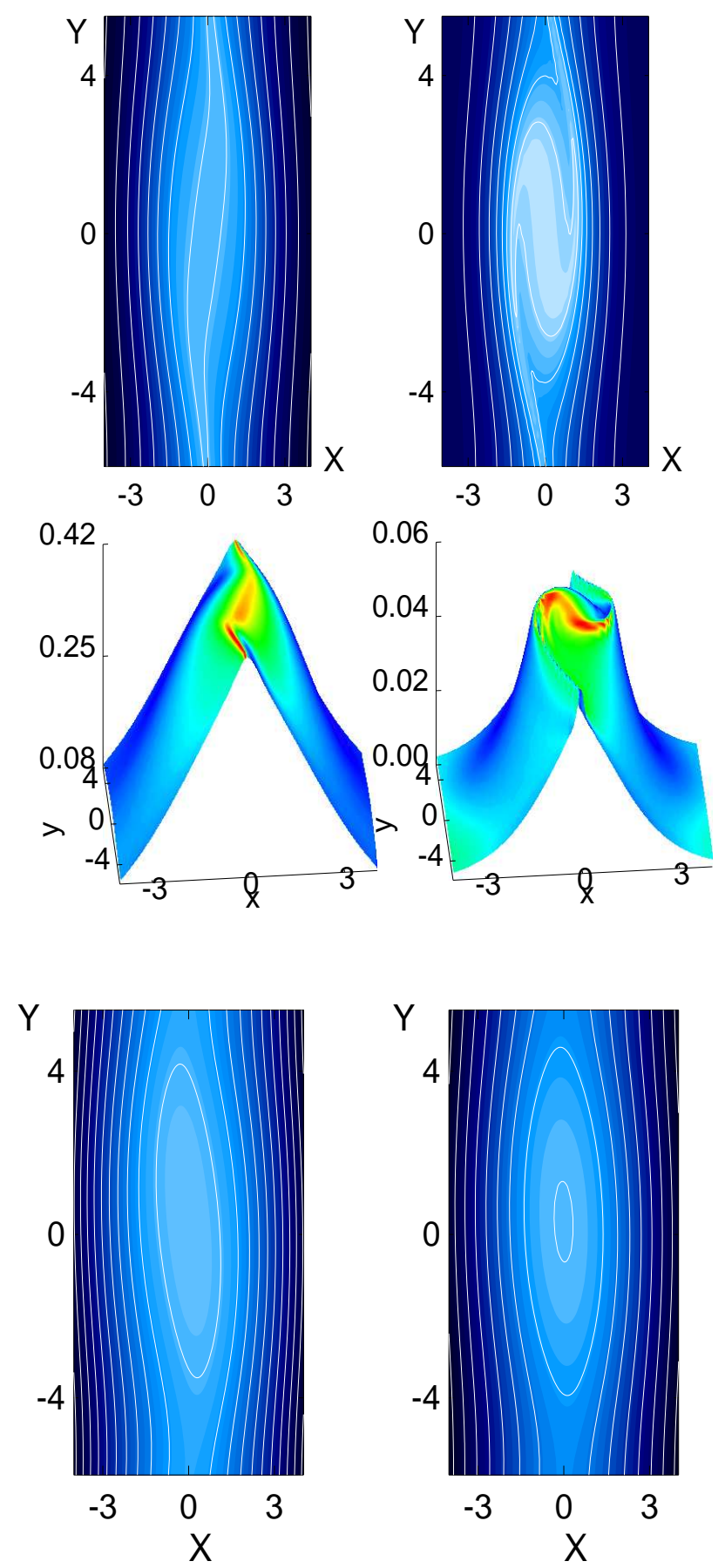

Fig. 5. Contour plots (top) and 3-D plots (middle) of the $p_{\|}=$constant foils of the electron distribution function and contour plots (bottom) of the kinetic stream function $\varphi_{k i n}$ at $t=81$ for $p_{\|}=0.5,1.5$ from left to right in the interval $-4<x<4$ around the neutral line. Note the different scales in the vertical axes of the 3-D plots.

growth is reached sooner. Here we present the results obtained in the case with $d_{e}=1$ and $v^{*}=4$. The evolution of the $\bar{p}_{\|}$-foils $f\left(x, y, \bar{p}_{\|}, t\right)$, restricted to the interval $-4<\mathrm{x}<4$ around the neutral line, is shown at $t=81$ for $\bar{p}_{\|}=-1.5,-0.5$ 
in Fig. (4) and for $\bar{p}_{\|}=0.5,1.5$ in Fig. (5), together with the contour plots of the stream function $\varphi_{k i n}$ in $\mathrm{x}-\mathrm{y}$ for the same values of $\bar{p}_{\|}$and the same interval in $\mathrm{x}$.

Foils corresponding to negative values of $\bar{p}_{\|}$were initially localized in two symmetric bands to the left and to the right of the neutral line and are thus modified by the onset of the reconnection instability only in their portion that extend into the reconnection region. On the contrary foils corresponding to positive values of $\bar{p}_{\| \mid}$were initially localized around $\mathrm{x}=0$ and are thus twisted by the development of the reconnection instability. The contour plots of the stream function $\varphi_{k i n}$ corresponds to a differential rotation in the $\mathrm{x}-\mathrm{y}$ plane. The sign of the rotation is opposite for positive and for negative values of $\bar{p}_{\|}$. The mixing caused by this differential rotation of the $p_{\|}$-foils is evident. As in the fluid case, the mixing of the Langrangian invariants in $\mathrm{x}-\mathrm{y}$ space is accompanied by the energy transfer towards increasingly small scales.

Within the range of parameters explored in the simulations discussed in the present paper, we have not evidenced any onset of a secondary instability. This result is fully consistent with the fluid simulations that show that the onset of the Kelvin-Helmholtz instability is impeded by increasing the electron temperature.

In summary, the Lagrangian invariants obtained by writing the drift-kinetic electron distribution function as a foliation of an infinite number of fluid type distributions taken at fixed parallel canonical momentum, lead us to establish a clear link between the fluid and the kinetic regimes of the reconnection instability since the (two) fluid invariants and the (infinite) drift-kinetic invariants evolve in time in an analogous fashion. In both cases the mixing of the Langrangian invariants in $x-y$ space and the energy transfer towards increasingly small scales make it possible for the system to reach a different macroscopic magnetic equilibrium with a saturated magnetic island and with a lower magnetic energy even in the absence of dissipation.

\section{Conclusions}

Magnetic field line reconnection in collisionless system is controlled by topological constraints that evolve in very similar forms in fluid and in kinetic regimes.

The form of these topological constraints depends explicitly on the microscopic physics that governs the reconnection process in the plasma. We find that the value of the microscopic parameters, such as the electron skin depth $d_{e}$ and the ion sound gyro-radius $\varrho_{s}$ in the fluid regimes, and the corresponding parameters in the drift kinetic regime, determine not only the linear growth rates of the reconnection instabilities, but also their characteristic nonlinear evolution times and, most important, the spatial distribution of the current and vorticity layers produced during the nonlinear development of the instabilities.

Indeed we find that, in the fluid regime, the ratio $\varrho_{s} / d_{e}$ determines a transition between a turbulent nonlinear regime, where long lasting fluid vortices develop inside a pulsating magnetic island, and a laminar regime with a (macroscopically) stationary island. In both regimes the current and vorticity layers eventually fill the entire magnetic island.

The topological constraints play an important role also in the kinetic regimes, as we have shown in the case of the drift kinetic model of the electron response for a two dimensional magnetic configuration with a strong guide field. In particular we have shown that the mixing of the Lagrangian invariants, the formation of smaller and smaller spatial scales and the energy transfer towards these increasingly small scales is common to the fluid and to the kinetic regimes of the Hamiltonian dynamics of a collisionless plasma and can be described in both regimes in analogous mathematical terms.

Acknowledgements. Part of this work was supported by the INFM Parallel Computing Initiative and by the Italian Ministry for University, by Scientific Research PRIN 2002 funds, by Russian Science Support Foundation and by Russian Foundation for Basic Research (Grant 04-01-00850).

Edited by: P.-L. Sulem

Reviewed by: two referees

\section{References}

Bhattacharjee, A., Ma, Z. W., Wang, X.: Recent developments in collisionless reconnection theory: Applications to laboratory, space plasmas,Phys. Plasmas, 8, 1829-1839, 2001.

Bergmans, J. and Schep, T. J.: Merging of Plasma Currents, Phys. Rev. Lett., 87, 195002-+, 2001.

Brunetti, M., Califano, F., and Pegoraro, F.: Asymptotic evolution of nonlinear Landau damping, Phys. Rev. E, 62, 4109-4114, 2000.

Cafaro, E., Grasso, D., Pegoraro, F., Porcelli, F., and Saluzzi, A.: Invariants, Geometric Structures in Nonlinear Hamiltonian Magnetic Reconnection, Phys. Rev. Lett.,, 80, 4430-4433, 1998.

de Blank, H. J.: Kinetic model of electrons in drift-Alfvén currentvortices, Physics of Plasmas, 8, 3927-3935, 2001.

de Blank, H. J. and Valori, G.: Electron kinetics in collisionless magnetic reconnection, Plasma Physics, Controlled Fusion, 45, A309-A324, 2003.

Del Sarto, D. Califano, F., and Pegoraro, F.: Secondary instabilities and vortex formation in collisionless-fluid magnetic reconnection, Phys. Rev. Lett., 91, 235001-+, 2003.

Fitzpatrick, R.: Scaling of forced magnetic reconnection in the Hallmagnetohydrodynamic Taylor problem, Phys. Plasmas, 11, 937946, 2004.

Fitzpatrick, R.: Scaling of forced magnetic reconnection in the Hallmagnetohydrodynamical Taylor problem with arbitrary guide field, Phys. Plasmas, 11, 3961-3968, 2004.

Grasso, D., Califano, F., and Pegoraro, F.: Ion Larmor Radius Effects in Collisionless Reconnection, Plasma Phys. Rep., 26, 512518, 2000.

Grasso, D., Califano, F., Pegoraro, F., and Porcelli, F.: Phase Mixing, Island Saturation in Hamiltonian Reconnection, Phys. Rev. Lett.,, 86, 5051-5054, 2001.

Harris, E. G.: On a plasma sheath separating regions of oppositely directed magnetic field, Il Nuovo Cimento, 23, 115-121, 1962. 
Kuvshinov, B. N., Pegoraro, F., and Schep, T. J.: Hamiltonian formulation of low-frequency, nonlinear plasma dynamics,Physics Letters A, 191, 296-300, 1994.

Kuvshinov, B. N., Lakhin, V. P., Pegoraro, F., and Schep, T. J.: Hamiltonian vortices and reconnection in a magnetized plasma, J. Plasma Physics, 59, 727-736, 1998.

Lancellotti, C., Dorning, J. J.: Nonlinear Landau Damping in a Collisionless Plasma, Phys. Rev. Lett., 80, 5236-+, 1998.

Lancellotti, C., Dorning, J. J.:Time-asymptotic wave propagation in collisionless plasmas, Phys. Rev. E, 68, 026406-+, 2003.

Liseikina, T. V., Pegoraro, F., and Echkina, E. Yu.: Phys. Plasmas, 11, 3535-3545, 2004.

Manfredi, G.: Long-Time Behavior of Nonlinear Landau Damping, Phys. Rev. Lett., 79, 2815-2818, 1997.

Morrison, P. J.: Hamiltonian formulation of reduced magnetohydrodynamics, Phys. Fluids, 27, 886-897, 1984.

Ottaviani, M. and Porcelli, F.: Nonlinear collisionless magnetic reconnection, Phys. Rev. Lett., 71, 3802-3805, 1993.

Ottaviani, M. and Porcelli, F.: Fast nonlinear magnetic reconnection, Phys. Plasmas, 2, 4104-4117, 1995.
Pegoraro, F.: Magnetic Field Line Reconnection in Dissipationless Regimes and Mixing of the Lagrangian Invariants in Strongly Magnetized, Two-dimensional, Plasma Configurations, in: Thematic Program in Partial Differential Equations, Workshop on Kinetic Theory, Fields Institute Toronto, http://www. fields.utoronto.ca/audio/\\#kinetic/, 2004.

Porcelli, F., Borgogno, D., Califano, F., Grasso, D., Ottaviani, M., and Pegoraro, F.: Recent advances in collisionless magnetic reconnection, Plasma Phys. Contr. Fusion, 44, B389 405, 2002.

Rogers, B. N., Denton, R. E., Drake, J. F., and Shay, M. A.: Role of Dispersive Waves in Collisionless Magnetic Reconnection, Phys. Rev. Lett., 87, 195004-+, 2001.

Schep, T. J., Pegoraro, F., and Kuvshinov, B. N.: Generalized twofluid theory of nonlinear magnetic structures, Physics of Plasmas, 1, 2843-2852, 1994.

Shay, M. A., Drake, J. F., Swisdak, M., and Rogers, B. N.: The scaling of embedded collisionless reconnection, Phys. Plasmas, 11, 2199-2213, 2004.

Valori, G.: Fluid, kinetic aspects of collisionless magnetic reconnection, ISBN 90-9015313-6, Print Partners Ipskamp, Enschede, the Netherlands, 2001.

Wang, X., Bhattacharjee, A., and Ma, Z. W.: Scaling of Collisionless Forced Reconnection,Phys. Rev. Lett., 87, 265003-+, 2001. 